\title{
scripted
}

Volume 16, Issue 1, August 2019

\section{Book review: Blockchain and the Law: The Rule of Code}

\author{
Primavera De Filippi and Aaron Wright \\ Cambridge, Massachusetts: Harvard University Press, 2018. 299 pages. ISBN \\ 978-0674976429. £25.95.
}

Reviewed by Joseph Savirimuthu*

\section{(c) (1) $(9$}

(C) 2019 Joseph Savirimuthu

Licensed under a Creative Commons Attribution-NonCommercial-

NoDerivatives 4.0 International (CC BY-NC-ND 4.0) license

DOI: 10.2966/scrip.160119.95

* Senior Lecturer in Law, Liverpool Law School, University of Liverpool, Liverpool, UK, jsaviri@liverpool.ac.uk 
Blockchains, distributed ledger technologies, bitcoins and peer-to-peer networks have reignited old debates and arguments about the implications of decentralisation for social, economic and political ordering. Blockchain and the Law: The Rule of Code sets out to map the landscape of this technology that enables its readers to assess the opportunities and regulatory challenges. The coverage is impressive, well-researched and key issues are examined and analysed with rigor and clarity. This book will appeal to technology and innovation scholars, policymakers and lawyers. It is also likely to be suitable for academic and policy programmes at undergraduate and postgraduate level. The book is wellstructured with an overriding goal - to define and explain the rationale and goals of the law in seeking to regulate the technology. As observers of the innovation opportunities and challenges will attest, the quest to formulate optimal strategies is complex and far from straightforward. It is a point that needs to be kept in mind that Blockchain and the Law will not resolve ongoing arguments about the mismatch between technological innovation and the ability of law to keep pace.

The book is structured with five Parts, beginning with an account of the characteristics of blockchain technology (Part 1) before proceeding to examine financial and contractual instruments (Part 2), security standards and protocols (Part 3), the institutional and organizational infrastructure (Part 4) and governance (Part 5). Whether the regulatory responses to blockchains will culminate in a lex cryptographica is merely a springboard for an examination of how the problems of associated with the automated and decentralised architecture are to be mitigated. For those with long memories of peer-to-peer file sharing services in the 1990s and 2000, one may need to be more circumspect about whether the distinctive decentralised software infrastructure upon which blockchain applications sit, will succeed in creating "order without law" (p. 5).

As noted above, blockchains are a particular form of peer-to-peer distributed ledger technology. Its exponential rise and transformative potential 
is acknowledged not only by innovators and business entrepreneurs but industries and governments in developed and developing economies. ${ }^{1}$ Chapters 1 and 2 provide readers with a good overview of key terminologies and characteristics of blockchain technologies and the myriad ways social, economic and legal relationships can be digitally constituted. Part 1 and the Introduction that precedes the detailed examination of blockchain applications lay the foundation to three limbs to the lex cryptographica thesis which frames the coverage of the book. First, the descriptive landscape of applications and uses of the distributed ledger system. Second, the practical and normative issues raised by this system of record and digital relationships. Third, institutional and regulatory challenges. The discussion of the technological aspects, particularly with the role of cryptography within blockchain applications is helpful to understanding not only evolving conceptions of trust and ownership but the range of digital interactions that can now be facilitated. An instance of how blockchain technologies require revisiting the rationales and goals for regulation can be found in the discussion relating to digital currencies and payment mechanisms in Chapters 3 and 5 respectively. Both chapters understandably emphasise the role and value of cryptography and distributed ledger technology in driving innovation in products and services, and contribute to reconceptualisations of trust, identity and authority. As the authors note, taking the case of the decentralisation of payment services:

'[w]hile blockchain offer[s] the promise of new and improved payment systems, the technology often runs into conflict with existing laws and

1 OECD, "OECD Blockchain Policy Forum - OECD”, available at http://www.oecd.org/finance/oecd-blockchain-policy-forum-2018.htm (accessed 3 December 2018). 
regulations because of its distributed, transnational, and pseudonymous nature.' (p. 65)

The implications of trust for contractual rules and the role of intermediaries in structuring supply chain networks and relationships from the basis of the discussion in Chapter 4. What are the serious doctrinal and practical ramifications stemming from platforms and applications leveraging blockchain technology through a combination of written agreements and automated processes (i.e. timestamps or tokens) that is regarded as signifying a "meeting of minds" or conclusion of the undertakings between the parties, without any human intervention? This chapter contains a useful treatment of these issues involved through the distinction made between "legal" and "smart" contracts while at the same time emphasising the nature of digital trust being shaped by the protocols embedded in the platforms. It is in this chapter that we also get a sense of not so much what lex cryptographica is but what the emergence of blockchain may imply for the invisible hand of lex mercatoria in guiding the evolution of standards and norms not necessarily tethered to orthodox conceptions of trustworthiness and the role of technological mechanisms for facilitating market interactions. ${ }^{2}$ The discussion also highlights some of the ways information asymmetries and trust implications can be addressed through the use of design and hybrid legal agreements (pp. 76-81) as well as mitigated in the context of the financial sector, concerns associated with an unregulated currency and ensuring systemic stability (pp. 98-100).

The implications of blockchains and distributed ledger technology for security, trust, identity and centralised authority which continue attract

2 Michele Finck, "Blockchains: Regulating the Unknown" (2018) 19 German Law Journal 665692. 
considerable interest are examined in Chapters 6 and 7. Issues such as inclusiveness, freedom from censorship and privacy are also sketched in these chapters. Readers wishing to learn more may find considerable literature which elaborates the deeper governance and practical issues. ${ }^{3}$ Both chapters suggest that notwithstanding the critical importance of managing cyber security risks, the role of blockchain technologies in aiding the development of tamper proof, resilience operationalisation and self-sovereign solutions is likely to be a limited one (pp. 107-127). It is important to note that dual-use technologies such as blockchains are not exceptional and that the opportunities made available by lex cryptographica also bring with it challenges in the form of social costs such as the sharing of copyrighted material and distribution of illegal content.

If blockchain technology can be seen as representing a cultural mindset towards the new matrix of wealth creation, along the lines eloquently set out by Yochai Benkler, where might this leave regulators and understandings of the way markets and economic organisations are being corporate world ${ }^{4}$ This is not merely a question about legal relations and mechanisms for decision-making but at the same time a political and ideological question and the authors do not

3 Primavera De Filippi, "Bitcoin: A Regulatory Nightmare to a Libertarian Dream" (2014) 3(2) Internet Policy Review, available at https://policyreview.info/articles/analysis/bitcoinregulatory-nightmare-libertarian-dream (accessed 3 December 2018); Primavera De Filippi and Raffaele Mauro, "Ethereum: The Decentralised Platform That Might Displace Today's Institutions" (Internet Policy Review, 25 August 2014), available at https://policyreview.info/articles/news/ethereum-decentralised-platform-might-displacetodays-institutions/318 (accessed 3 December 2018); Primavera De Filippi and Benjamin Loveluck, "The Invisible Politics of Bitcoin: Governance Crisis of a Decentralised Infrastructure" (2016) 5(3) Internet Policy Review, available at https://policyreview.info/articles/analysis/invisible-politics-bitcoin-governance-crisisdecentralised-infrastructure (accessed 3 December 2018).

4 Yochai Benkler, The Wealth of Networks: How Social Production Transforms Markets and Freedom (New Haven: Yale University Press, 2006). 
attempt to provide a comprehensive analysis of the complexities. ${ }^{5}$ The discussion in the next three chapters (Chapters 8-10) provides a compressed reflection of the impact of blockchain technology on the social costs of organisation. Not surprisingly, Ronald Coase's seminal paper provides the backdrop for an appraisal of the distributive, stewardship and managerial transformations blockchains potentially make possible. ${ }^{6}$ Indeed, the invisible hand is an apt mechanism for allocating resources, why do firms exist? The argument made by Coase was that the organisation of firms mirrors the goal of individuals wishing to reduce the transaction costs such as those associated with search, bargaining, and entering into agreements. Why do blockchain exist, one could ask? These chapters throw some light on the novel nature of the regulatory challenges posed by blockchain technologies - they are not centralised and do not rely on hierarchical structures of authority or formalised decision-making mechanisms. The discussion in these chapters gives us a glimpse of the paradigm shift that is likely to take place in the light of what we have already seen in the way Facebook and Uber now leverage the decentralised architecture of platform infrastructures. Blockchain technologies, the authors argue, allow us to extend Coase's transaction costs to "decrease uncertainty among shareholders" (p.136), transform decision making processes within organisations (p. 137-140) and create new avenues for raising investment. ${ }^{7}$ The opportunities and the regulatory challenges decentralised, automated organisations pose for securities, investment, limited liability and more broadly the orthodox business model are

5 Mark Fenwick, Wulf Kaal, and Erik Vermeulen, "Why ‘Blockchain' Will Disrupt Corporate Organizations" (2018), available at http://papers.ssrn.com/abstract=3227933 (accessed 3 December 2018).

6 Ronald Coase, "The Nature of the Firm" (1937) 4 Economica 386-405.

7 Aurelio Gurrea-Martínez and Nydia Remolina, "The Law and Finance of Initial Coin Offerings" (2018), available at http://papers.ssrn.com/abstract=3182261 (accessed 3 December 2018). 
explored in Chapters 8 and 9. Smart sensors, intelligent devices and ready availability of platform infrastructures and the cloud are leading to a reduction of transaction costs in the traditional way of doing business and contributing to the decentralisation of market interactions and exchanges. Chapter 9 which is titled "The Blockchain of Things" provides some further reflections on the new wave of changes to traditional business models and the responses needed from regulators to support these innovations in services and functionalities. One is inclined to agree with Arthur Clarke, that should these innovations come pass, the boundaries between advanced technology and magic would be indistinguishable. ${ }^{8}$ For the moment, we can say that blockchain and the internet of things are not magic. However, the issues highlighted in these chapters are profound. The potential of decentralised infrastructures to enhance efficiencies, transparency and trust are evident as shown in discussions on Decentralized Autonomous Organizations ("D Corp"), Initial Coin Offerings and Tokens. Issues raised by the D Corp Hack, the egalitarian underpinnings of blockchain and cryptocurrency technologies would not be out of place here or in the chapters discussing the modes of governance. Is blockchain hashing a new approach to new forms of collaboration, crowdfunding and open governance? Chapters 11, 12 and the conclusion contain some of the most interesting conceptual and normative accounts in respects of the blurring of rules of codes and the rule of law. Lessig and leading scholars, it will be recalled, in framing technology as instruments of standard-setting, decision-making and behaviour-modification alerted us to the normative tensions embedded in technological infrastructures. These chapters extend the insights and considerable debates revolving around the "Code is Law" thesis. I am not too sure that we will live in a world dominated

8 Arthur Clarke, Profiles of the Future: An Inquiry into the Limits of the Possible (New York: Harper and Row, 1973). 
by a lex cryptographica and the authors too seem like-minded (pp. 203-204, 207210). ${ }^{9}$ What Yochai Benkler alluded to in The Wealth of Networks is equally true of the likely reach (and limits) of lex cryptographica as it has been the case with the Internet and derivative innovations - that there are no spaces of perfect freedom from all constraints (p. 161).

Blockchain and the Law is an excellent book. Whether one agrees with the thesis, one thing is certain: Its mapping of the technical landscape, intellectual rigour and vision of a technologically infused society will undoubtedly generate renewed enthusiasm for inter-disciplinary scholarship. That said, there is a regulatory blindspot couched in lex cryptographica and Satoshi Nakamoto's progeny that is easily obscured. It is still worth keeping in mind that some values are not easily captured by code and the rule of law's appeal lies in its enduring ability to respond to tyrannies in both human and algorithmic forms.

9 De Filippi and Loveluck, supra n. 3. 